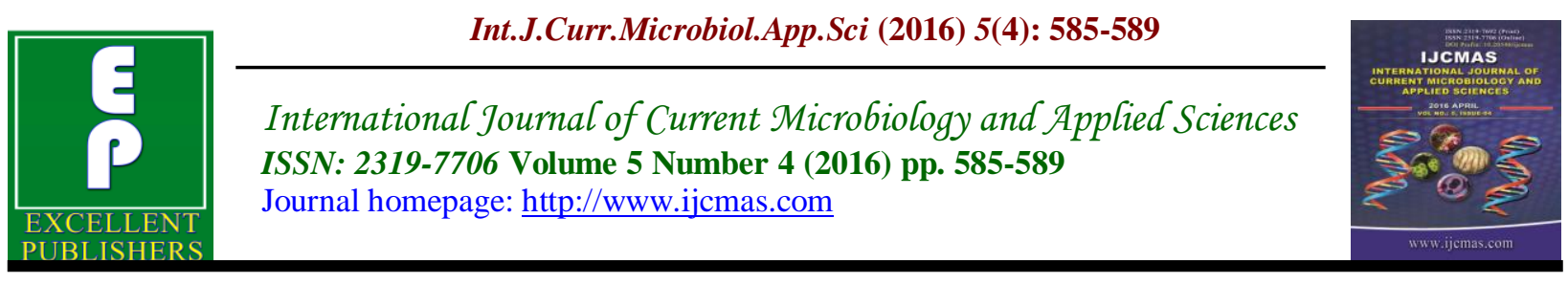

Original Research Article

http://dx.doi.org/10.20546/ijcmas.2016.504.066

\title{
Association between Uric acid and HbA1c in type 2 Diabetes Mellitus in Comparison with Controls
}

\author{
V. Pavithra, K. Revathy and S. Swaminathan* \\ Department of Biochemistry, Apollo Speciality Hospitals, Ayanambakkam, Chennai, India \\ *Corresponding author
}

\begin{tabular}{ll}
\hline & A B S T R A C T \\
\cline { 2 - 2 } $\begin{array}{l}\text { Key w ord s } \\
\text { UA, }\end{array}$ & $\begin{array}{l}\text { Previous studies have predicated an association between UA, the end product of } \\
\text { purine metabolism to T2DM as it is said to induce hyper insulinemia and impaired } \\
\text { glucose tolerance. UA is also associated with the alterations of other organ function } \\
\text { HbA1c, }\end{array}$ \\
CVD. & $\begin{array}{l}\text { such as Kidney and Cardiac and elevated UA has been implicated in a number of } \\
\text { other complications of T2DM such as neuropathy, obesity, hypertension and } \\
\text { metabolic disorders. This study was undertaken to find out the association between }\end{array}$ \\
\hline Article Info & $\begin{array}{l}\text { UA and HbA1c in T2DM in comparison with controls. Very good correlations } \\
\text { (p<0.0001) were found out between patients \& controls for both UA and HbA1c } \\
\text { Accepted: } \\
\text { 19 March 2016 }\end{array}$ \\
$\begin{array}{l}\text { Available Online: } \\
\text { to April 2016 }\end{array}$ & $\begin{array}{l}\text { large number of patients and to include UA as one of the tests to be done for T2DM } \\
\text { patients. }\end{array}$
\end{tabular}

\section{Introduction}

Type2 Diabetes Mellitus (T2DM) is prevalent in a majority of population in India and approximately $30-35 \%$ of Indian populations are living with this syndrome. Many previous studies have linked Uric Acid (UA) to DM and some associations have been found out between them. As glycosylated Hemoglobin (HbA1c) is now routinely used to monitor diabetic control, this study was undertaken to find out if any association exists between UA and $\mathrm{HbAlc}$ in T2DM patients.

UA is associated with metabolic, cardiovascular and renal abnormalities in T2DM patients but is less well understood in T1DM.
Glycosuria rather than hyperglycemia increases uricosuria in T1DM. Future studies should examine the effect of UAlowering therapies to assess the impact of ambient glycemia, which causes an important uricosuric effect (Lytvyn et al., 2015). Conflicting data exist about UA levels in T2DM, as low levels were found in some T2DM patients, while elevated serum UA is a feature of hyperinsulinemia and impaired glucose tolerance. In T2DM, hyperuricemia seems to be associated with the insulin-resistant syndrome and with early onset or increased progression to overt nephropathy, while hypouricemia is associated with worse metabolic control, hyperfiltration and a late onset or decreased progression to overt nephropathy (Simona 
bo, et al., 2001). The incident rates of diabetes and prediabetes with Insulin Resistance (IR) and Impaired Fasting Glucose (IFG) were higher among persons with greater serum UA concentrations. Hyperuricemia in the mid-twenties is an independent marker for predicting diabetes and prediabetes among young adults in the subsequent 15 years (Eswar Krishnan et al., 2012).

Administration of UA and vitamin C selectively improved acetylcholine responses in patients with T1DM and in regular smokers with T2DM. UA administration improved endothelial function in the forearm vascular of patients with T1DM and smokers, suggesting that high UA level in vivo might serve as protective role in these and other conditions associated with increased cardiovascular risk (Stephen Waring et al., 2006).

The relationship between elevated UA and Coronary Heart Disease (CHD) is discussed controversially. The Prevalence of hyperuricaemia was dependent on age and duration of the disease in T1DM patients, whereas in recently diagnosed T2DM patients the prevalence of elevated UA levels were higher than in patients with long-standing T1DM, without any further increase with longer duration of the disease. An elevated UA level was also associated with Body Mass Index(BMI), hypertension and nephropathy in both types of diabetes and in both sexes. In women, hyperuricaemia was correlated with the presence of CHD both in T1DM and T2DM. The exclusion of hypertension and nephropathy in the multiple logistic regression analysis had no effect on these associations. However, after adjustment for these two factors, a significant correlation between hyperuricaemia and CHD was found in T2DM diabetic men. In addition, increased serum UA was associated with gangrene in male T2DM patients. These results suggest that elevated UA levels shows correlation with the presence of CHD in females rather than in male diabetic patients, independently of hypertension and nephropathy (Rathmann et al., 1993).

InT2DM patients serum UA level early in the course of diabetes and independent of confounders is significantly associated with later development of persistent macro albuminuria. Therefore, UA may be a novel and important player in the pathogenesis of microvascular complications in diabetes. A dose-response relationship between UA and early decline in renal function has recently been demonstrated in patients with T1DM. Randomized controlled trials on drugs that lower UA need to be conducted to evaluate the causal relationship between UA and development and progression of diabetic kidney disease; in addition, large scale longterm treatment trials need to be performed, as they are still lacking (Peter Hovind et al., 2011).

UA is increased in patients with T2DM with neuropathy versus those without. Whether UA is involved in the pathogenesis of T2DM peripheral neuropathy remains to be established (Papanas, et al., 2011). The association between UA level and risk for hypertension and IFG or T2DM was stronger among men with a $\mathrm{BMI}<24.2$ $\mathrm{kg} / \mathrm{m}^{2}$ than among men with a BMI $\geq 24.2$ $\mathrm{kg} / \mathrm{m}^{2}$, although the absolute risk was more greater in obese men. These results indicate that UA level is closely associated with an increased risk for hypertension and IFG in T2DM patients (Nakanishi et al., 2003). Recent studies have introduced UA as a potential risk factor for developing diabetes, hypertension, stroke and Cardio Vascular Diseases. The value of elevated levels of UA in serum as a risk factor 
for diabetes development is still under scrutiny. Recent data suggest that UA clearance is being reduced with an increase in IR and hence UA may serve as a marker of prediabetes. With aging, UA increases in serum of diabetic patients and this effect is more profound in males than in females diabetics. Since literature data suggest a strong genetic effect on UA levels, it would be pertinent to perform further, possibly genetic studies, in order to clarify gender and ethnic differences in UA concentrations (Causevic et al., 2010).

\section{Materials and Methods}

25 male and female patients and 50 controls in the age group of 45-75 yearswere selected for this study. For controls, patients attendingthe Master Health Checkupwere enrolled and for patients,those attending the Diabetic Clinic were selected. The main objective of this study was to find out the association between HbA1c and Uric Acid between Controls and Patients.

Diuri CS 1300 B analyser and Dialab reagents were used to measure Uric Acid and Biorad D10 analyser and the kit supplied by that company was used to measure HbA1c. The accuracy of these analytes were validated by the use of BioRad accuracy controls at two levels.

\section{Inclusion Criteria}

Patients who attended the Endocrine Clinic and whose $\mathrm{HbA} 1 \mathrm{c}>7.5 \%$ were included.

Patients who attended the routine Master Health Checkup and whose HbA1c and Uric Acid levels were within normal range served as controls.

\section{Exclusion Criteria}

Patients who attended the Diabetic Clinic and whoseHbA1c values $<7.5 \%$ were excluded.

For statistical analysis of data, a software downloaded from the website http://www.graphpadquickcalcs.comwas used to calculate, students ' $t$ ' distribution ( $t$ ) and probability $(\mathrm{p})$ between the group of analytes studied for both controls and between controls and patients.

\section{Results and Discussion}

Table I presents the mean\& SD results for all Patients\& Controls together with such results for Male and Female populations. It is clear from this Table that mean results for HbA1c\&UA are higher for patients compared to controls and this Table gives some preliminary opinion about the association that may exist between HbA1c and UA.

Table II shows the statistical parameters ( $\mathrm{t}$ and $\mathrm{p}$ ) between Patients and Controls for the analytes studied. Very good association were observed between Patients \& Controls for both $\mathrm{HbA1c} \& \mathrm{UA}(\mathrm{P}<0.001)$ but only a moderate correlation $(\mathrm{P}=0.0637)$ for female patients.

Many previous studies have linked UA to T2DM, but studies linking UA to HbA1c are scarse. Some studies have observed an increase in UA levels in T2DM patients and our study has now found out a quantitative relationship between $\mathrm{UA}$ and $\mathrm{HbA1c}$, the diabetic control marker. Increased UA have been observed in patients with T2DM, but there was no experimental data available and this study has proved good association between them. Previous studies have established some significant correlation between hyperuricemia and CHD linked to T2DM in Diabetic men which are in agreement with our study (5). 
Table.1 Mean and SD for all Patient \& Controls

\begin{tabular}{|c|c|c|c|c|c|c|}
\hline \multirow{2}{*}{ S.No } & \multirow{2}{*}{ Patient groups } & \multirow{2}{*}{$\mathbf{n}$} & \multicolumn{2}{|c|}{ HbA1c } & \multicolumn{2}{|c|}{ UA } \\
\hline & & & Mean & SD & Mean & SD \\
\hline \multicolumn{7}{|c|}{ Controls } \\
\hline 1 & All patients & 50 & 5.37 & 0.357 & 5.028 & 1.338 \\
\hline 2 & Males & 25 & 5.38 & 0.294 & 5.624 & 1.176 \\
\hline 3 & Females & 25 & 5.36 & 0.416 & 4.432 & 1.238 \\
\hline \multicolumn{7}{|c|}{ Patients } \\
\hline 1 & All patients & 50 & 9.66 & 1.542 & 3.954 & 1.119 \\
\hline 2 & Males & 25 & 9.86 & 1.498 & 4.136 & 0.999 \\
\hline 3 & Females & 25 & 9.46 & 1.589 & 3.772 & 1.220 \\
\hline
\end{tabular}

Table.2 Statistical Parameters ( $t$ and $p$ )

\begin{tabular}{|c|l|l|l|l|}
\hline \multirow{2}{*}{ S.No } & \multicolumn{1}{|c|}{ Group } & \multicolumn{1}{|c|}{$\begin{array}{c}\text { Analytes } \\
\text { Compared }\end{array}$} & \multicolumn{1}{|c|}{$\mathbf{t}$} & \multicolumn{1}{|c|}{$\mathbf{P}$} \\
\hline 1 & $\begin{array}{l}\text { Controls Vs Patients } \\
(\mathrm{n}=50)\end{array}$ & HbA1c & 19.1695 & $<0.0001$ \\
\cline { 2 - 5 } & Uric Acid & 4.3534 & $<0.0001$ \\
\hline 2 & $\begin{array}{l}\text { Controls Vs Patients } \\
(\text { Males ;n=25) }\end{array}$ & HbA1c & 14.667 & $<0.0001$ \\
\cline { 3 - 5 } & Uric Acid & 4.8209 & $<0.0001$ \\
\hline 3 & $\begin{array}{l}\text { Controls Vs Patients } \\
(\text { Females ;n=25) }\end{array}$ & HbA1c & 12.4843 & $<0.0001$ \\
\cline { 3 - 5 } & Uric Acid & 1.8982 & 0.0637 \\
\hline
\end{tabular}

In conclusion, Based on our understanding from previous studies, many of which did not establish quantitative association between UA and $\mathrm{HbAlc}$, this study has strongly established an association between UA and HbA1c $(\mathrm{P}<0.0001)$ there by linking UA, the end product of purine metabolism to DM, particularly T2DM. This study was done only on T2DM patients. The outcome of this study will enable future researchers to extend this by including more patients and to recommend the inclusion of UA measurement along with Diabetic profile tests for T2DM patients.

\section{Acknowledgement}

The authors would like to thank Dr.Mitra Ghosh, Chief of Lab Services at Apollo Speciality Hospital, Ayanambakkam,
Chennai, TamilNadu for giving us permission to undertake this study.

\section{References}

Causevic, A., Semiz, S., Macic Dzankovic, A., Cico, B., Dujic, T., Malenica, M., Bego TBosn, J. 2010. Relevance of uric Acid in progression of type 2 diabetes mellitus. Basic Med. Sci., 10(1): 54-9.

Eswar Krishnan, Bhavik J. Pandya, Lorinda Chung, Ali Hariri, Omar Dabbous. 2012. Hyperuricemia in Young Adults and Risk of Insulin Resistance, Prediabetes and Diabetes: A 15-Year Follow-up Study. Am. J. Epidemiol., 176(2): 108-16.

Lytvyn, Y., Škrtić, M., Yang, G.K., Yip, P.M., Perkins, B.A., Cherney, D.Z. 
2015.

Glycosuria-mediated urinary uric acid excretion in patients with uncomplicated type 1 diabetes mellitus. Am. J. Physiol. Renal. Physiol., 308(2): F77-83.

Nakanishi, N., Okamoto, M., Yoshida, H., Matsuo, Y., Suzuki, K., Ta, K. 2003. Serum uric acid and risk for development of hypertension and impaired fasting glucose or Type II diabetes in Japanese male office workers. European J. Epidemiol., 18(6): 523-530.

Papanas, N., Katsiki, N., Papatheodorou, K., Demetriou, M., Papazoglou, D., Gioka, T., Maltezos, E. 2011. Peripheral Neuropathy is Associated with Increased Serum Levels of Uric Acid in Type 2 Diabetes Mellitus. Angiol., 62(4): 291-5.

Peter Hovind, D., Peter Rossing, Richard, J., Johnson, Hans-Henrik Parving. 2011. Serum Uric Acid as a New Player in the Development of Diabetic Nephropathy. J. Ren. Nutr., 21(1): 124-7.

Rathmann, W., Hauner, H., Dannehl, K., Gries, F.A. 1993. Association of elevated serum uric acid with coronary heart disease in diabetes mellitus. Diabete Metabolisme, 19(12): $159-166$.

Simona, bo., Cavallo-Perin, P., Luigi, Gentile, Gianfranco Pagano. 2001. Hypouricemia and hyperuricemia in type 2 diabetes: Two different phenotypes. European J. Clin. Investigation, 31(4): 318-21.

Stephen Waring, W., John, A., McKnight, David, J., Webb, Simon, R.J., Maxwell. 2006. Uric Acid Restores Endothelial Function in Patients with Type 1 Diabetes and Regular Smokers. Diabetes, 55(11): 3127-32.

\section{How to cite this article:}

Pavithra, V., K. Revathy and Swaminathan, S. 2016. Association between Uric acid and $\mathrm{HbAlc}$ in type 2 Diabetes Mellitus in Comparison with Controls. Int.J.Curr.Microbiol.App.Sci. 5(4): 585-589. doi: http://dx.doi.org/10.20546/ijcmas.2016.504.066 